\section{FARADIC STIMULATION OF NERVE AND MUSCLE DURING OPERATIONS.}

BY HARRY PLATT, M.S. LOND., F.R.C.S. ENG.,

CAPTAIN, R.A.M.C. (T.); HON. SURGEON AND SURGEON IN CHARGE, ORTHOPADIC DEPARTMENT, ANCOATS HOSPITAL, MANOHESTER ORTHOPADIC SURGEON IN CHARGE, SPECIAL MILITARY SURGICAI SECTION, 2ND WESTERN GENERAL HOSPITAL; AND

E. S. BRENTNALL, M.B. MANOH., F.R.C.S. EDIN., ASSISTANT SURGEON, SPECIAL MUITARY SURGICAL SECTION, 2ND WESTERN GENERAL HOSPITAL; MEDICAL OFFICER IN CHARGK, MASSAGE AND ELECTRO-THERAPEUTIOAL DEPARTMLNT ANCOATS HOSPITAL, MANCHESTER.

IN the operative exploration of peripheral nerve trunks direct faradic stimulation of the exposed nerve is an essential feature in an efficient technique. The information thus supplied, is of a comprehensive nature. Not only is it possible to determine the presence or absence of conductivity in the nerve above and below the lesion, but, in addition, details of intraneural topography can be investigated, and this is of especial importance in dealing with lesions which involve a part only of the cross section of a nerve trunk. When several nerve trunks are exposed at the same time, in a limb in which the normal anatomical landmarks have been lost owing to extensive scarring and destruction of muscle tissue, faradic excitation often provides a ready means of identifying each nerve. These facts are, perhaps, recognised by the majority of surgeons engaged in reconstructive surgery and need no further elaboration.

Value of Identification of Motor Branches of Nerve Trunks.

In our experience of 340 operations on peripheral nerves we have been impressed by the extreme importance of the exact recognition of the point of origin and distribution of muscular branches arising from nerve trunks in the limits of the operation field. The use of faradic stimulation renders possible the identification of each branch exposed and the confirmation of its physiological integrity. Such information is invaluable.

The classical anatomical text-book descriptions of the number and position of the motor branches of nerve trunks can be tested, if necessary, amplified or totally refuted-a true example of the investigation of the anatomy of the living-and in an end-to-end suture operation a record of its exact site can be made with reference to the motor branch proximal and distal to the line of suture. The accuracy of the future clinical examinations of a recovering nerve depends to a considerable extent on an authoritative record of the operation findings, and particularly on a knowledge of the relation of the level of the lesion and its repair to motor branches which have been identified.

The anatomical or physiological integrity of muscular branches may be endangered in the course of an operation, either by accident, which implies carelessness or a lack of knowledge of anatomical minutiæ, or with deliberate intent. End-to-end-suture in extensive lesions is achieved only after free longitudinal exposure of the nerve trunk, and this often demands considerable displacement of, and tension on, muscular branches; this is especially marked when a nerve trunk is displaced far from its normal bed-e.g., in the well-known manœuvre of displacement of the ulnar nerve in front of the internal epicondyle. In a few cases, even after this procedure, the gap is so great that end-to-end apposition can be effected only after division of one or more branches proximal to the gap. By the physiological experiment of direct stimulation of these muscular branches a scientific control is provided; the choice of the correct branch for sacrifice and the conservation of the remaining branches is in this way ensured.

Observations of Practical and Scientific Interest.

The relation of muscular branches to isolated "adhesions" between nerve trunks and muscle bellies, and to the sprouting masses of nerve fibrils which are found binding nerves to muscles, has both a practical and scientific interest. several occasions we have identified motor branches in what appeared to be isolated adhesions, and we would emphasise the value of stimulating such tissue before freeing a nerve widely. At the same time the extensive perineural adhesions encountered at levels where motor branches are unknown and presenting the physical appearance designated as "nerve callus" (Cone) are invariably silent on direct stimulation. In our earlier operative work on nerve injuries the possibility of the occurrence of spontaneous neurotisation of a muscle belly by the outgrowth of axons into the muscle fibres after an injury to a nerve trunk was considered, in view of the suggestive experimental work of Heineke, Erlacher, and Steindler, ${ }^{1}$ on the neurotisation of paralysed muscles. The negative results of the stimulation of neuro-muscular adhesions in a large number of operations have caused us to rule out the possibility of this phenomenon.

In addition to observations on the conductivity in a nerve trunk and its branches, the direct response of the muscle bellies exposed in the wound is to be noted; this includes muscles supplied by nerves other than the one presenting a lesion. Intramuscular and perimuscular scarring and actual loss of muscle substance accompany peripheral nerve injuries in so many cases that the response or lack of response to faradic stimulation of a given muscle is information which helps to complete an adequate conception of the physiological and pathological anatomy of the tissues explored.

So far we have dealt with the mere presence or absence of response in nerve and muscle. A quantitative estimation of the results of stimulation is not easily arrived at, but using the technique described below, we have evolved a rough personal comparative standard of nerve and muscle response as the result of careful observations on a large series of cases. In this connexion we have found that in nerve lesions presenting the clinical syndrome of causalgia, excepting those in which a sufficiently gross intraneural lesion has produced a block in a motor fasciculus, the nerve trunk and the muscles supplied by it, both above and below the lesion, show a striking degree of hyper-excitability.

In operations for infantile paralysis the use of faradic stimulation is equally valuable, and the information gained as to the exact response in muscles which are to be used or discarded in such operations as tendon transplantation or tenodesis is complementary to that derived from clinical examinations and the conventional external electrical tests.

$$
\text { Teohnique of Faradic Stimulation. }
$$

We have used the bipolar method in preference to the unipolar method, the outstanding objections to the latter being the tenaiency to diffusion of the current and the inconvenience of using an indifferent pad electrode, which must be fixed and maintained in position outside the sterile operation area. On the other hand, bipolar stimulation, employing fine-pointed electrodes closely approximated, permits extreme accuracy in the localisation of the stimulus-an essential feature in the investigation of nerve topography and in dealing with fine motor branches.

A simple bipolar instrument consists of two small surgical probes sharpened down to fine points at one end, covered with rubber tubing, except at the extremities, and bound together by a few turns of linen thread, with a small wooden wedge intervening to ensure stability. Rubber-covered rheophores are fixed by binding screws to the proximal ends of the probes, which are bent in an outward direction. The whole apparatus thus assembled is sterilised by boiling, dried between sterile towels - an important step-and is then ready for use. During the application of the stimulus the electrode is held by a Lane's forceps or other form of clamp.

For the provision of the current a Bristow faradic coil serves admirably, the strength of current used being minimal. As the variations in intensity from time to time are probably small and the resistance of the electrode in a dry wound fairly constant, with repeated observations a comparative standard of nerve and muscle response can be developed.

In conclusion, we would emphasise the great value of observations made in the fullest detail on the response of nerve and muscle tissue to direct faradic stimulation at the time of exploratory operations. The information thus gained cannot be acquired with certainty, or in many instances is not available from any other method of investigation. 1 Heineke: Centralblatt f. Chir., 1914, xli. Erlacher: Zeitschrift f.
Orthop.. Chir., xxxlv.; Amer. Journ. Orthop. Surg., July, 1915. Orthop.e Chir., xxxlv.; Amer. Journ. Orthop.

Dr. Saint René Bonnet has resumed his thermal practice at Chatel-Guyon-les-Bains, Auvergne, France, on demobilisation from the French Army. 